\title{
Empirical treatment with fibrinolysis and early surgery reduces the duration of hospitalization in pleural sepsis
}

\author{
T.K. Lim, N.K. Chin
}

\begin{abstract}
Empirical treatment with fibrinolysis and early surgery reduces the duration of hospitalization in pleural sepsis. T.K. Lim, N.K. Chin. C ERS Journals Ltd 1999.

ABSTRACT: The efficacy of three different treatment protocols was compared: 1) simple chest tube drainage (Drain); 2) adjunctive intrapleural streptokinase (IP-SK); and 3) an aggressive empirical approach incorporating SK and early surgical drainage (SK+early OP) in patients with pleural empyema and high-risk parapneumonic effusions.

This was a nonrandomized, prospective, controlled time series study of 82 consecutive patients with community-acquired empyema $(n=68)$ and high-risk parapneumonic effusions $(n=14)$. The following three treatment protocols were administered in sequence over 6 years: 1) Drain $(n=29$, chest catheter drainage); 2) IP-SK $(n=23$, adjunctive intrapleural fibrinolysis with $\left.250,000 \mathrm{U} \cdot \mathrm{day}^{-1} \mathrm{SK}\right)$; and 3) SK+early OP $(n=30$, early surgical drainage was offered to patients who failed to respond promptly following initial drainage plus SK).

The average duration of hospital stay in the SK+early OP group was significantly shorter than in the Drain and IP-SK groups. The mortality rate was also significantly lower in the SK+early OP than the Drain groups (3 versus $24 \%$ ).

It was concluded that an empirical treatment strategy which combines adjunctive intrapleural fibrinolysis with early surgical intervention results in shorter hospital stays and may reduce mortality in patients with pleural sepsis. Eur Respir J 1999; 13: 514-518.
\end{abstract}

Dept of Medicine, National University Hospital, Singapore.

Correspondence: T.K. Lim

Dept of Medicine

National University Hospital

Lower Kent Ridge Road

Singapore

Singapore 119074

Fax: 657794112

Keywords: Fibrinolysis parapneumonic effusion pleural empyema streptokinase thoracoscopy thoracotomy

Received: August 111998

Accepted after revision November 281998
Pleural empyema is a serious complication of pleuropulmonary sepsis. It can be associated with considerable morbidity and a mortality rate $>20 \%$ [1-3]. It usually develops as a parapneumonic effusion which occurs in up to $40 \%$ of patients with community-acquired pneumonia [4]. A sub-group of patients acquire high-risk parapneumonic effusions that progress rapidly from free-flowing pleural exudates to multiloculated fibrinopurulent collections within a few days [4-6]. Pleural empyema may also appear de novo without an associated pneumonic process.

The treatment for pleural sepsis involves empirical antibiotics for the most prevalent bacteria plus expedient and safe drainage of the infected pleural cavity. There are various methods of draining the pleural cavity. These include needle aspiration, chest tube drainage, thoracoscopic drainage and open thoracotomy. Recent advances in this area include the use of smaller, flexible image-guided catheters [7], the administration of adjunctive intrapleural fibrinolytic agents to facilitate fluid drainage [8], and surgical interventions such as video-assisted thoracoscopic drainage (which is less painful than conventional thoracotomy) $[9,10]$.

The clinical efficacy of adjunctive fibrinolysis using streptokinase (SK) had been evaluated in two controlled trials $[11,12]$. Both studies reported that significantly larger volumes of pleural fluid were drained from patients treated with daily intrapleural instillations of SK than from control patients. DAVIES et al. [11] also showed that the treatment group had better lung expansion at the time of discharge from hospital. However, it has also been shown that the administration of SK did not result in significant improvement in the key measures of clinical outcome such as duration of hospital stay, the need for surgical drainage or mortality [12]. Nevertheless, intrapleural SK is relatively safe [13], inexpensive and may induce prompt resolution in some patients [12]. Thus, it may be offered as an adjunctive modality in the initial empirical treatment of pleural sepsis [14].

WAIT et al. [15], in a randomized controlled trial of patients with loculated parapneumonic empyema, found that a strategy of primary treatment by video-assisted thoracoscopic surgery was associated with higher efficacy, shorter hospital stay and lower cost than intrapleural streptokinase [15]. This high success rate of early surgical intervention is consistent with the experiences of POTHULA and Krellenstein [16], Lemense et al. [17] and Thourani et al. [18] all of whom employed more the invasive thoracotomy.

An aggressive approach to pleural sepsis that incorporates the recent advances of image-guided placement of drains, intrapleural fibrinolysis and early surgical drainage has been advocated by both S.A. Sahn [17] and R.W. Light $[19,20]$. This recommendation of an early aggressive approach to surgical drainage is based, however, mostly upon expert opinion and not on evidence derived from large comparative studies. In this regard, DAVIES and GLEESON [21] suggested that large numbers of patients should be enrolled into multicentre controlled trials. 
Therefore, in this controlled study, the efficacy of an aggressive, empirical approach which incorporated pleural fibrinolysis with early surgical drainage in the treatment of pleural sepsis was examined.

\section{Methods}

This was a prospective, controlled, time-series study of three different treatment protocols in 82 consecutive patients admitted to the hospital with community acquired pleural empyema and high-risk parapneumonic effusions from 1990 to 1997 [22]. Patients with malignant effusions, tuberculous or fungal empyema, post-traumatic, postoperative and hospital-acquired pleural sepsis were excluded. Empyema [23] was defined as 1) distinctly purulent fluid, 2) positive bacterial culture from the fluid and/or 3) positive Gram stain for bacteria. High-risk parapneumonic effusion [23] was defined as 1) $\mathrm{pH}<7$ and/or 2) lactate dehydrogenase $(\mathrm{LDH})$ level $>1,000 \mathrm{U} \cdot \mathrm{L}^{-1}$.

Diagnostic thoracenthesis with a $14-\mathrm{G}$ venula was performed for all patients in whom pleural sepsis was suspected. The pleural fluid samples were sent for differential cell counts, biochemical analysis, Gram stain and bacterial cultures. Following the diagnosis of either empyema or high-risk parapneumonic effusion, closed-tube thoracenthesis was attempted for all patients. At the bedside, size 24 chest tubes were inserted into patients with large, dependent pleural fluid collections while smaller 7 to $12-$ French pigtail catheters were placed under image guidance in the radiology department for those with multiloculated or nondependent fluid collections. All patients were assessed at least once daily and the need for further imaging and drainage was decided on an individual basis.

The three treatment protocols were instituted in seuence. Simple tube drainage (Drain) was utilized as the primary drainage modality in 29 patients, adjunctive intrapleural streptokinase (IP-SK) was administered in 23 patients and 30 patients were treated with an empirical approach which incorporated SK and early surgical drainage (SK+early OP). The same group of chest physicians managed all patients during the study period and they followed a consistent approach to the schedule of initial empirical antibiotics, decisions on the efficacy of treatment and fitness for hospital discharge.

In the IP-SK protocol, 250,000 U SK with $100 \mathrm{~mL}$ saline was instilled into the pleural cavity on a daily basis. The SK was left in the pleural cavity for about $4 \mathrm{~h}$ following which the chest tube was unclamped, and the fluid was manually aspirated and left to drain passively into a water-sealed chamber.

In the SK+early OP protocol, the patients were reviewed soon after each aspiration of SK fluid and, based upon the volume of fluid aspirated, a decision was made as to whether a second dose of SK was indicated. The intention was, in patients who did not respond satisfactorily to the first dose of SK, to repeat a dose within $24 \mathrm{~h}$. This follows the suggestion of MOuLTON et al. [8] that a better result might be achieved by administering more than one dose of SK in the same day.

In the Drain and IP-SK protocols, surgical drainage was performed only when the pleural sepsis had obviously failed to respond, with persistence or exacerbation of the septic state (temperature and blood leukocyte counts) and pleural fluid accumulation. In the SK+early OP protocol, surgical drainage was offered to the patients within 48-72 $\mathrm{h}$ after the first dose of SK if they failed to show an adequate response to the initial empirical treatment. Adequate response was defined as: 1) volume of fluid drainage commensurate with the estimated size of the pleural collections; 2) definite improvement in radiological features (either on plain radiographs or computed tomography); 3 ) resolution of fever (a febrile response induced by intrapleural SK usually lasts $<12 \mathrm{~h}$ ); and 4) improvement of leukocytosis. This aggressive empirical approach is consistent with the recommendations of most experts [17, $19,20]$. Thoracotomy was the most common $(>80 \%)$ surgical drainage procedure performed in this study.

The results are expressed as mean (SD) values. Continuous variables were compared with paired and unpaired Student's t-tests where appropriate while the Chi-squared test was used to test for differences between proportions. The efficacy of the three treatment protocols was evaluated on a prospective, "intention to treat" basis. Data from the Drain and IP-SK treatment groups have been described in an earlier publication [12].

\section{Results}

There were 82 patients ( 65 males and 17 females) with a mean (SD) age of 57 (17) yrs (range 16-88 yrs). Most patients had some comorbidity, either diabetes mellitus or chronic cardiorespiratory illness. There were no significant differences in age, sex ratio, frequency of comorbidity and severity of peripheral leukocytosis between the three groups of patients (table 1).

There were no significant differences between the study groups in the frequency of distinct empyema, bacterial isolates, pleural leukocytosis and biochemistry (table 2). There was, however, a significantly larger number of locules detected (per patient) in the SK+early OP group (table 2) than in the other two groups.

The bacteriological results are shown in table 3 . The frequencies of positive pleural fluid isolates were comparable between the three groups of patients. However, there were proportionately larger numbers of patients with polymicrobial and Gram negative infections in the Drain compared to the SK+early OP groups.

Table 1. - Patient characteristics

\begin{tabular}{lccc}
\hline & \multicolumn{3}{c}{ Treatment groups } \\
\cline { 2 - 4 } & Drain & IP-SK & SK+early OP \\
\hline Patients n $^{+} 29$ & 23 & 30 \\
Age $^{+}$yr & $63(14)$ & $50(19)$ & $57(15)$ \\
Male $/$ Female $_{\text {Comorbidity* \% }}$ & $23 / 6$ & $18 / 5$ & $26 / 4$ \\
TWBC $\times 10^{6} \cdot \mathrm{L}^{-1}$ & 16210 & 65 & 56 \\
\hline
\end{tabular}

${ }^{+}$: Means (SD). Drain: simple chest tube drainage; IP-SK: adjunctive intrapleural streptokinase; SK+early OP: streptokinase + early surgical drainage. $*$ : frequency of underlying diabetes mellitus, chronic cardiorespiratory illness, end-staged renal failure and cancer. TWBC: total white blood cell count. There were no statistically significant differences between the three groups of patients. 
Table 2. - Pleural fluid characteristics

\begin{tabular}{lccc}
\hline & \multicolumn{3}{c}{ Treatment groups } \\
\cline { 2 - 4 } & Drain & IP-SK & SK+early OP \\
\hline Empyema $^{+} \%$ & 79 & 74 & 80 \\
Locules $^{*} \mathrm{n}$ & $1.1(0.3)$ & $1.3(0.6)$ & $1.7(0.9)^{*}$ \\
$\mathrm{pH}$ & $7.0(0.6)$ & $7.2(0.2)$ & $7.1(0.3)$ \\
$\mathrm{TWBC} \times 10^{6} \cdot \mathrm{L}^{-1}$ & 8300 & 5700 & 15400 \\
$\mathrm{LDH} \mathrm{U} \cdot \mathrm{L}^{-1}$ & 16700 & 14850 & 16000 \\
\hline
\end{tabular}

Number of locules and $\mathrm{pH}$ data presented as mean (SD). For treatment group definitions see table $1 .^{+}$: percentage of patients with empyema as defined in the Methods section; ${ }^{*}$ : the number of distinct pleural collections per patient. TWBC: total white blood cell count; LDH: lactate dehydrogenase level. *: $\mathrm{p}<0.01$, patients in the SK+early OP group versus the other two groups.

Table 4 compares the intensity of treatment and timing of surgical drainage between the study groups. Patients in the IP-SK treatment group received the most chest tubes and were administered significantly more doses of SK than those in the SK+early OP group. No adverse effects could be ascribed to SK treatment. Surgical drainage (thoracotomy $>80 \%$ ) was performed in a larger proportion of patients in the SK+early OP group than either Drain (statistically significant, $\chi^{2} \mathrm{p}<0.05$ ) or IP-SK (NS) groups. Surgical intervention was instituted an average of 12 days earlier in the SK+early OP group than in the other two study groups. This data confirm that there was overall adherence to the strategy of early, aggressive and complete surgical drainage in the SK+early OP protocol. No major complications were noted following surgery and no patient required a repeat operation.

Table 5 compares the key outcome measures between the study groups. The duration of hospital stay was approximately one week less in patients treated according to the SK+early OP protocol than those managed according to the other two protocols. Furthermore, the mortality rate in the SK+early OP group was significantly lower than in patients treated with simple tube drainage (Drain group). Seven patients in the Drain group died, six from septicaemia and one following a myocardial infarction. The majority of deaths occurred in the first week of hospitalization and involved elderly males with comorbid diseases. Two patients in the SK group died, one from septicaemia and the other from myocardial infarction. The single death in the SK+early OP group occurred in a patient suffering from alcoholism who developed systemic sepsis and acute respiratory distress syndrome following successful surgical drainage of the empyema.

\section{Discussion}

This was a prospective, controlled study of the efficacy of combining intrapleural fibrinolysis with early surgical drainage in the treatment of community-acquired pleural sepsis. It confirms the results of investigators who had previously reported success rates of up to $95 \%$ with early aggressive surgical intervention $[16,17]$. It is also consistent with the findings of WAIT et al. [15] who, in a small but well conducted and randomized trial, showed that primary treatment with video-assisted thoracoscopic drainage was more cost-effective than conventional medical drainage with adjunctive fibrinolysis. THOURANI et al. [18], in a
Table 3. - Bacterial cultures in the pleural fluid and blood

\begin{tabular}{lccc}
\hline & Drain & IP-SK & SK+early OP \\
\hline Aerobic Gram-positive cocci & 7 & 5 & 6 \\
Staphylococus aureus & 5 & 2 & 4 \\
Streptococcus pneumoniae & - & 2 & 1 \\
Other streptococcus species & 2 & - & 1 \\
Enterococcus faecalis & - & 1 & - \\
Aerobic Gram negative bacilli & 8 & 4 & 1 \\
Klebsiella pneumoniae & 4 & 4 & 1 \\
Pseudomonas aeruginosa & 1 & - & - \\
Escherichia coli & 2 & - & - \\
Enterobacter species & 1 & - & - \\
Anaerobes & 5 & 4 & 4 \\
Bacteroides & 3 & 2 & 1 \\
Streptococcus milleria & 1 & 2 & 2 \\
Peptostreptococcus & 1 & - & 1 \\
Positive blood cultures & 2 & 1 & 0 \\
\hline
\end{tabular}

The number of patients with positive pleural fluid cultures were comparable between the three groups: 59\% (Drain), 48\% (IPSK) and $37 \%$ (SK+early OP). However, polymicrobial pleural isolates were noted only in $20 \%$ of the Drain and IP-SK groups but not the SK+early OP group. Streptococcus was isolated in blood cultures of three patients. For treatment group definitions see table 1 .

retrospective cost-effectiveness analysis, also concluded that early surgical treatment of multiloculated empyemas with decortication resulted in reduced charges owing to shortened length of stay and decreases in the number of radiological procedures performed. Furthermore, a significant reduction in mortality rate was observed when this aggressive empirical approach was compared with conventional chest catheter drainage (table 4). Thus, it was confirmed that a treatment strategy which incorporates many of the recent advances in pleural fluid drainage can have a positive impact on the clinical outcome of pleural sepsis.

The limitations of this study are the lack of randomization, and that the investigators were not blinded and did not use preset criteria for decision making. However, all consecutive patients were studied, and they were managed in a consistent manner in the same centre and by a small group of physicians. The three treatment protocols were instituted over consecutive epochs and the analysis completed on an "intention to treat" basis [14, 22]. Moreover, the characteristics of the study groups, including frequency of distinct empyema and positive bacterial cultures were comparable (tables 1, 2 and 3). In particular, anaerobic [24] and viridans streptococcal [1] bacterial infections which have been associated with poorer outcomes in pleural sepsis, were present in similar frequencies in the three groups of patients (table 3). There was also no change in the pattern of antibiotics prescribed for community-acquired pneumonia and pleural sepsis during the study period.

If there was any element of bias in this study it would most likely have occurred during administration of the IPSK protocol. The authors were enthusiastic about pleural fibrinolysis and, based upon uncontrolled reports, had anticipated a positive outcome with SK therapy. This was also reflected in the intensity of treatment where the largest numbers of chest catheters per patient were administered in the IP-SK group (table 4). Despite good adherence to the IP-SK protocol and close patient review however, no 
Table 4. - Intensity of treatment and timings of surgical drainage

\begin{tabular}{|c|c|c|c|}
\hline & \multicolumn{3}{|c|}{ Treatment groups } \\
\hline & Drain & IP-SK & SK+early OP \\
\hline 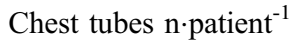 & $1(0.8)$ & $1.8(1.2)^{* *}$ & $1(0.8)$ \\
\hline $\begin{array}{l}\text { Streptokinase } \\
\text { dose } \text { patient }^{-1}\end{array}$ & - & $5.1(2.5)^{\#}$ & $2.5(1.5)$ \\
\hline $\begin{array}{l}\text { Days before surgical } \\
\text { drainage }\end{array}$ & $19.5(12)$ & $19(5)$ & $7(5)^{+}$ \\
\hline Surgical intervention $\%$ & 14 & 22 & 43 \\
\hline
\end{tabular}

Data presented as mean (SD) or percentage where indicated. For definition of treatment groups see table 1. *: from start of hospitalization. ${ }^{* *}: \mathrm{p}<0.01$, versus Drain and $\mathrm{SK}+$ early OP groups; ${ }^{\#}: \mathrm{p}<0.01$, versus $\mathrm{SK}+$ early OP group; ${ }^{+}: \mathrm{p}<0.01$, versus the other two groups.

significant effect was found on the duration of hospital stay, the need for surgical drainage, and mortality, when compared to drainage with chest tubes alone (table 5).

Because only a small number of patients were recruited, a clinically important effect of SK could have been missed in the present study [19]. Furthermore, there was a lower (statistically insignificant) mortality rate in the SK group compared to the Drain group. Intrapleural SK is relatively inexpensive and safe. Moreover, a large number of uncontrolled studies have reported high success rates with fibrinolysis and a few of the present patients (including some with multiloculated empyemas) did respond rapidly and completely to adjunctive SK $[12,19]$. The possibility, suggested by MouLTON et al. [8] that achieving a more intense pleural fibrinolysis by administering more than one dose of SK per day might be more effective was considered. This was incorporated into the SK+early OP protocol. However, only three patients $(10 \%)$ in the SK+early OP group received more that a single dose of SK per day. Thus, it was not possible to assess the efficacy of a more intensive pleural fibrinolysis regimen. This strategy may merit a separate study.

Despite uncertainty about the efficacy of pleural fibrinolysis in pleural sepsis, a trial of intrapleural SK may be appropriate for initial empirical treatment. Failure of the pleural sepsis to respond adequately (as defined in Methods) in 48-72 h however, may be an indication for more aggressive intervention [14]. Since SK itself may increase the net volume of pleural fluid, increased volume of pleural fluid drainage following adjunctive SK therapy should be interpreted with caution [25]. It should not be the only justification for prolonged tube drainage and repeat doses of SK. An early decision on the need for further intervention was likely a key element in the success of the SK+early OP protocol (tables 4 and 5). Surgical drainage was performed, on average, 12 days earlier in the SK+early OP group and was the main factor responsible for the shorter duration of hospital stay. These results thus corroborate the report by СНАм et al. [26] that showed that late surgical referral is an important problem in the treatment of pleural empyema.

Standard thoracotomy is a major surgical procedure. It is associated with considerable pain and a prolonged postoperative recovery phase. In the traditional management of pleural sepsis, most physicians only resort to surgical intervention when patients have clearly failed to respond to
Table 5. - Clinical outcomes

\begin{tabular}{lccc}
\hline & \multicolumn{3}{c}{ Treatment groups } \\
\cline { 2 - 4 } & Drain & IP-SK & SK+early OP \\
\hline LOS $^{+}$days & $21.4(12)$ & $22.2(11)$ & $15.1(5)^{* *}$ \\
Mortality \% & 24 & 9 & $3^{\#}$ \\
\hline
\end{tabular}

Data are presented as mean (SD) or percentage where indicated. For definition of treatment groups see table $1 .^{+}$: Length of hospital stay (LOS) (as in the previous report [12], two patients in the Drain group who had prolonged stays in hospital for reasons unrelated to pleural sepsis were excluded). $* *: p<0.01$, versus the other two patient groups; ${ }^{\#}: \mathrm{p}<0.01$, versus the Drain group.

more conservative drainage procedures. This was the approach taken during the Drain and IP-SK protocols of this study. It resulted in average hospitalizations of approximately 3 weeks (table 4). This is comparable with the median duration of hospitalization of 23 days reported in a recent prospective, multicentre study from the UK [2]. It is also similar to the experience of THOURANI et al. [18] who reported an average stay of 22 days in patients treated initially with image-directed catheter drainage.

Video-assisted thoracoscopic surgery is an important advance in the surgical treatment of pleural sepsis $[9,10]$. It is a less invasive procedure than thoracotomy and yet highly effective in achieving pleural debridement and clearance. Only four (18\% of surgical drainage) patients were treated with thoracoscopy in this study, two each in the IP-SK and SK+early OP groups. It was therefore not possible to compare directly the results of thoracoscopic versus thoracotomic drainage. WAIT et al. [15] showed, however, that early application of video-assisted thoracoscopic drainage can reduce the length of hospitalization to well below 2 weeks. The cost-efficacy of an intermediate drainage procedure such as medical thorascopy, which obviates the need for general anaesthesia, also requires further evaluation [27].

The significantly lower mortality in the SK+early OP group than in the Drain group of patients is another notable result of this study (table 4). The mortality rate in the Drain group is, however, in the higher range of that in most other reports. In view of the small number of patients studied, higher than usual mortality experienced by the Drain group and the lack of prospective randomization, the observation that the SK+early OP protocol had a positive impact on mortality should be interpreted with caution. The patients in the Drain group were older and had more comorbid diseases than those in the SK+early OP group although these differences were not statistically significant. The Drain group, however, had more complex empyemas with a significantly higher number of pleural locules than the SK+early OP group. Overall, the two groups of patients had comparable prognostic features.

Septicaemia was an important cause of death in the Drain group. It is doubtful if early surgical intervention can have a positive impact on mortality from overwhelming sepsis in elderly patients with comorbid diseases. The relative contributions of an intensive adjunctive SK regimen and early surgical drainage in reducing mortality in this study also requires further investigation.

In conclusion, the treatment of pleural sepsis, an empirical approach that incorporates adjunctive fibrinolysis 
and early aggressive surgical drainage may reduce the duration of hospital stay. More prospective studies are needed to evaluate the real impact of recent advances in pleural fluid drainage on morbidity and mortality. Future studies will need to compare different approaches in patient groups stratified according to a well-defined classification scheme $[4,19]$.

\section{References}

1. Jerng JS, Hsuch PO, Teng LJ, Lee LN, Yang PC, Luh KT. Empyema thoracis and lung abscess caused by viridans streptococci. Am J Respir Crit Care Med 1997; 156: $1508-1514$.

2. Ferguson AD, Prescott RJ, Selkon JB, et al. The clinical course and management of empyema. $Q J$ Med 1996; 89: 285-289.

3. Kelly JW, Morris MJ. Empyema thoracis: medical aspects of evaluation and treatment. South Med J 1994; 87: 11031110 .

4. Light RW, Girard WM, Jenkinson SG, George RB. Parapneumonic effusions. Am J Med 1980; 69: 507-512.

5. Light RW. A new classification of parapneumonic effusions and empyema. Chest 1995; 108: 299-301.

6. Heffner JE, Brown LK, Barberi C, DeLeo JM. Pleural fluid chemical analysis in parapneumonic effusions: a meta-analysis. Am J Respir Crit Care Med 1995; 151: $1700-1708$

7. Ulmer JL, Choplin RH, Reed JC. Image-guided catheter drainage of the infected pleural space. J Thorac Imaging 1991; 6: 65-73.

8. Moulton JS, Berikert RE, Weiseiger KH, et al. Treatment of complicated pleural fluid collections with image guided drainage and intra cavitary urokinase. Chest 1995; 108: 1252-1259.

9. Ridley PD, Baimbridge MV. Thoracoscopic debridgement and pleural irrigation in the management of empyema thoracis. Ann Thorac Surg 1991; 51: 461-464.

10. Landreneau RJ, Keenan RJ, Hazelring SR, et al. Thoracoscopy for empyema and hemothorax. Chest 1995; 109: 18-24.

11. Davies RJO, Traill ZC, Gleeson FV. Randomized controlled trail of intrapleural streptokinase in community acquired pleural infection. Thorax 1997; 52: 416-421.
12. Chin NK, Lim TK. Controlled trial of intrapleural streptokinase in the treatment of pleural empyema and complicated parapneumonic effusions. Chest 1997; 111: 275-279.

13. Davies CWH, Lok S, Davies RJO. The systemic fibrinolytic activity of intrapleural streptokinase. Am J Respir Crit Care Med 1998; 157: 328-330.

14. Lim TK, Chin NK. Streptokinase for empyema. Lancet 1997; 350: 446.

15. Wait MA, Sharma S, Hohn J, Nogare AD. A randomized trial of empyema therapy. Chest 1997; 111: 1548-1551.

16. Pothula V, Krellenstein DJ. Early aggressive surgical management of parapneumonic empyema. Chest 1994; 105: 832-836.

17. LeMense GP, Strange C, Sahn SA. Empyema thoracis: therapeutic management and outcome. Chest 1995; 107: 1532-1537.

18. Thourani VH, Brady KM, Mansour KA, Miller JI, Lee RB. Evaluation of treatment modalities for thoracic empyema: a cost effective analysis. Ann Thoracic Surg 1998; 66: 1121-1127.

19. Hamm H, Light RW. Parapneumonic effusion and empyema. Eur Respir J 1997; 10: 1150-1156.

20. Light RW. The management of parapneumonic effusions and empyema. Curr Opin Pulmon Med 1998; 4: 227-229.

21. Davies RJO, Gleeson FV. The diagnosis and management of pleural empyema. Curr Opin Infect Dis 1998; 11: 163168.

22. Chin NK, Lim TK. Treatment of complicated parapneumonic effusions and pleural empyema: a four-year prospective study. Singapore Med J 1996; 37: 631-635.

23. Light RW. Pleural Diseases. 3rd Edition. Baltimore, Williams \& Wilkins, 1990; pp. 129-153.

24. Bartlett JG. Anaerobic bacterial infections of the lung and pleural space. Clin Infect Dis 1993; 16 (Suppl. 4): S248S255.

25. Strange C, Allen AL, Harley R, et al. Intrapleural streptokinase in experimental empyema. Am Rev Respir Dis 1993; 147: 962-966.

26. Cham CW, Haq SM, Rahamin J. Empyema thoracis: a problem with late referal? Thorax 1993; 48: 925-927.

27. Loddenkemper R, Schonfeld N. Medical thoracoscopy. Curr Opin Pulmon Med 1998; 4: 230-234. 